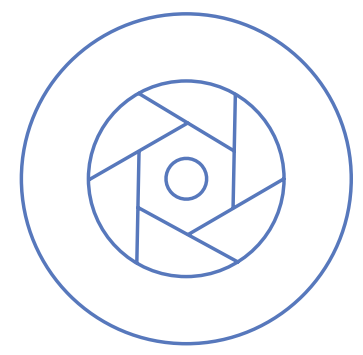

Care for women includes women having adequate access to food and health care to prevent illness, availability of fertility regulation and birth spacing options, sufficient time for rest, and protection from abuse

Ann Nutr Metab 2020;76(suppl 3):36-49

\title{
Maternal Undernutrition before and during Pregnancy and Offspring Health and Development
}

\author{
Melissa F. Young and Usha Ramakrishnan
}

Key Insights

Maternal undernutrition remains a critical public health problem with large regional and intra-country disparities in the prevalence of underweight, anemia, and micronutrient deficiencies. The greatest burden is seen among the poorest women in poor countries. While the obesity epidemic is growing, the persistence of underweight in some countries in South Asia and Africa remains unacceptably high. Another major problem that disproportionately affects women of reproductive age is anemia, which is also associated with an increased risk of poor maternal and infant outcomes. A key driver of poor nutrition is food insecurity. Despite the existence of evidence-based strategies for improving maternal nutrition during pregnancy, there are still large gaps in program implementation and outreach.

\section{Current knowledge}

Globally, $9.7 \%$ of women are underweight and $14.9 \%$ are obese. The nutritional and health status of women as they enter pregnancy is known to play a key role in placental function and the subsequent growth and development of the fetus. The placenta regulates nutrient availability for fetal growth and ultimately influences the long-term health of the newborn. Micronutrients, including iron, zinc, folic acid, and other vitamins, contribute to genome-wide alterations and/or epigenetic modifications during the critical period of organogenesis. These changes influence subsequent outcomes, such as body composition, metabolism, immunity, and cognitive function, in the offspring.

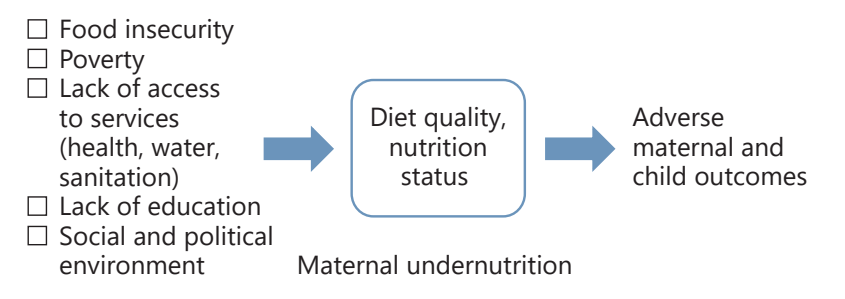

Maternal undernutrition is highly prevalent among the poorest women and is driven by a complex series of factors.

\section{Practical implications}

At the political level, women's nutrition has not received sufficient prioritization. Furthermore, restricting the focus of women's nutrition to pregnancy places a significant limit on the effectiveness of interventions. For women who live in conditions of extreme food insecurity (with anemia, micronutrient deficiencies, and undernutrition), maternal nutrition interventions during pregnancy may arrive too late. We urgently need to reach women earlier, in order to provide preconception care and family planning services. Programs focused on school-aged or adolescent girls have been identified as promising strategies in this context. Future efforts need to ensure that programs reach vulnerable and marginalized communities in order to address regional disparities in maternal undernutrition.

\section{Recommended reading}

Black RE, Victora CG, Walker SP, Bhutta ZA, Christian P, de Onis $M$, et al.; Maternal and Child Nutrition Study Group. Maternal and child undernutrition and overweight in low-income and middle-income countries. Lancet. 2013;382(9890):427-51. 